\title{
OMASES: A DYNAMIC SECURITY ASSESSMENT TOOL FOR THE NEW MARKET ENVIRONMENT
}

\author{
A. BIHAIN D. CIRIO M. FIORINA R. LOPEZ D. LUCARELLA S. MASSUCCO D. RUIZ VEGA C. VOURNAS \\ T. VAN \\ L. WEHENKEL \\ Tractebel University \\ CESI \\ Alstom \\ CESI \\ (I) \\ University of \\ University of \\ ICCS/NTUA \\ CUTSEM \\ (B) of Genoa (I) (I) $\quad$ T\&D (F) \\ Genoa (I) Liege (B) \\ (G) \\ University of \\ Liege (B) \\ University of \\ Liege (B)
}

\section{OMASES Working Group:}

A. Bihain (Tractebel, B), G. Burt (University of Strathclyde, UK), G. Christoforides (HTSO, GR), D. Cirio (University of Genova, I), A. Danelli (CESI, I), B. Delfino (University of Genova, I), G. Demartini (CESI, I), M. Fiorina (CESI, I), S. Galloway (University of Strathclyde, UK), J. Giri (Alstom T\&D, USA), G. Hasse (University of Liege, B), N. Hatziargyriou (National Technical University of Athens, GR), JD. Georgiadis (National Technical University of Athens, GR). J. Kabouris (HTSO, GR), T. Koronides (HTSO, GR), R. Lopez (Alstom T\&D, F), G. Manos (National Technical University of Athens, GR), S. Massucco (University of Genova, I), J.R. Mc Donald (University of Strathclyde, UK), A. Morini (University of Genova, I), M. Pavella (University of Liege, B), D. RuizVega (University of Liege, B), L. Signoretty (Alstom T\&D, F), T. Siewierski (University of Strathclyde, UK), F. Silvestro (University of Genova, I), M. Stubbe (Tractebel, B), J. Taisne (Alstom T\&D, F), T. Van Cutsem (University of Liege, B), G. Vimercati (CESI, I), C. Vournas (National Technical University of Athens, GR), L. Wehenkel (University of Liege, B).

\begin{abstract}
The paper presents the efforts and results of a large consortium of European Industries, Research Centers and Universities involved in an EU research project named OMASES in the field of Power System Dynamic Security Assessment (DSA).

The overall structure of an on-line DSA tool including TSA Transient Stability Assessment, VSA - Voltage Stability Assessment, TS - Training Simulator and MS - Market Simulator is reported. Some basic assumptions and methodological aspects of the tool are presented considering its possible use within actual or future Energy Management Systems under the new electric market environment. Scenarios set up for the validation phase and results are reported with reference to the Italian test facility.
\end{abstract}

Keywords -- Power system security - Dynamic security assessment - Transient stability - Voltage stability - Operator training simulator - Market simulator - Transmission capacity

\section{INTRODUCTION}

$\mathrm{T}$ HE liberalization of electricity market is posing great challenges to the operation of interconnected power systems [1]. The necessity of permitting open access to power producers and to allow large quantity of power flowing along the transmission grid require advanced capacity of monitoring and controlling security limits. The changes in responsibilities for overall system reliability particularly for what concerns the property of generators and tools for maintaining it, are expected to result in more stressed power systems. Dynamic security may become more critic than static security, thus it will be necessary to analyze and simulate possible contingencies in order to classify potential harmful situations and to indicate and even operate preventive and/or corrective remedial actions.
The paper presents the overall structure and the on-going results of a research project partially supported by the European Union within the aims of the Framework VEnergy scheme. The project is named OMASES - Open Market Access and Security Assessment System [2]. The OMASES Consortium includes Industries, Research Centers, Universities and System Operators.

OMASES scope is to provide Energy Management System (EMS) operators with a tool for Dynamic Security Assessment (DSA) to be used on-line during the normal cycle of real-time operation, in operational planning and as a dispatcher training simulator including a simulation of the deregulated electric market environment. DSA is realized through different application functions: TSA - Transient Stability Assessment, VSA - Voltage Stability Assessment, TS - Training Simulator and MS - Market Simulator.

The OMASES technical aims are: to provide a transparent methodology to assess the network dynamic stability, to increase the power flow on electrical lines by computing dynamically operating limits, to reduce the lines incidents resulting from stability problems by simulating dynamic contingencies, to improve the generation allocation by computing the unit commitment in accordance with the dynamic limits and the electrical market demand, to adjust emergency protection to more accurate values either off line or on line (according to field equipment capabilities), and to provide advanced real time dynamic simulation tool for operator training.

The following sections of the paper report the OMASES hardware and software architecture, some theoretical bases and the hypothesis under which the application functions have been chosen and adapted to OMASES goals, and the experimental sites that are used for testing the tool. The 
paper will particularly focus on the Italian experimental site and will provide information on the individuated scenarios to be used to test the DSA functions included in OMASES. The second experimental site, located at the Greek TSO (HTSO), is addressed in another paper [3].

\section{DSA SYSTEM REQUIREMENTS AND OMASES ARCHITECTURE}

\section{II.a) - Fundamental DSA Requirements}

In a synthetic sentence, OMASES is a tool including some application functions relevant to DSA phenomena such as TSA and VSA, making use of a powerful training simulator, including the market and capable of proposing preventive remedial actions. OMASES can be inserted in existing EMS or engineered into new EMS structure. The following modes can be activated: (1) Engineering mode, (2) Real-time mode, (3) Training mode. A short description of each mode is reported below.

(1) Engineering mode: it is the off-line application of TSA and VSA studies involving or not the market environment and performed mainly for planning purposes

(2) Real-time mode: the EMS feeds OMASES with network data and solutions. DSA is synchronized with data transfer and cyclically runs with an overall execution time within 15 minutes

(3) Training mode: the operator gets used with the DSA tools and power system dynamics, performs analysis (future scenarios, post-event analysis, etc.) of the existing electrical system and/or experiments. In this mode, market rules can be used to realistically affect on the operational stage.

TSA and VSA are quite mature methodologies and techniques at least in the Engineering mode. Efforts have been done [2], [4], [5] to use them in the Real-time mode and to include them into realistic Training mode. TS and MS are very much related to existing power system simulator and to specific market. Therefore, within OMASES, the MS is intended to provide credible scenarios for subsequent TS and DSA activities.

\section{II.b) - OMASES Architecture}

The OMASES applications run in a distributed environment, with a link to the host EMS over which a real-time picture of the power network is obtained. The architecture includes a data server which will host any shared information, database systems, message systems, etc.; an appropriate server (even in parallel computing configuration) for each Application Function (TSA, VSA, MS, TS); computer systems to be used by operation personnel for user interface processes; a LAN structure for inter-machine communications allowing easy integration also in existing EMS. The global frame that will constitute the DSA environment is shown in Figure 1 [6].

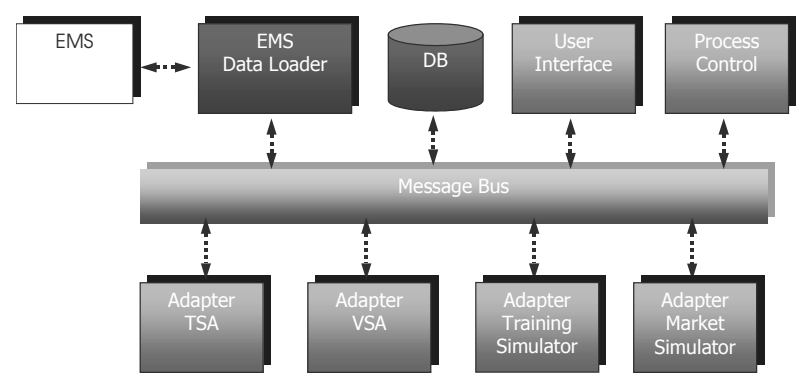

Fig. 1. Overall OMASES architecture and Application Functions

\section{OMASES APPLICATION FUNCTIONS}

Both TSA and VSA Application Functions need to systematically investigate a large number of contingencies, at least all the credible ones. Contingency number around one thousand is not unusual in large interconnected systems. This implies a contingency filtering and ranking that allow to extract a subset of contingencies that may really affect system security. Advanced DSA tools further than identifying potentially harmful situations, also provide, for these types of contingencies, preventive remedial actions [7]. Each of the TSA and VSA functions calculates system security indices and margins for a set of contingencies applied on a real time scenario directly taken from the EMS (real-time mode) or a simulated one generated by the TS (training mode).

\section{III.a) - TSA Transient Stability Assessment and Control}

The theoretical backgrounds and the current state of TSA and Control (TSA\&C) make use of the SIME method, a tool that has been developed at the University of Liege [7], [8]. SIME is a hybrid transient stability method that can use detailed models of power system components and control devices available in the most advanced codes for power system simulation. The filtering/ranking capability of TSA is synthetically shown in the following Figure 2 that refers to a specific case reported in [7] with number between brackets expressing the contingencies examined and filtered. Stability/Instability indices (such as CCT Critical Clearing Time) are computed by using the principle of the Equal Area Criterion and by reducing the overall system multi-machine dynamics to a single machine-infinite bus (OMIB) dynamics.

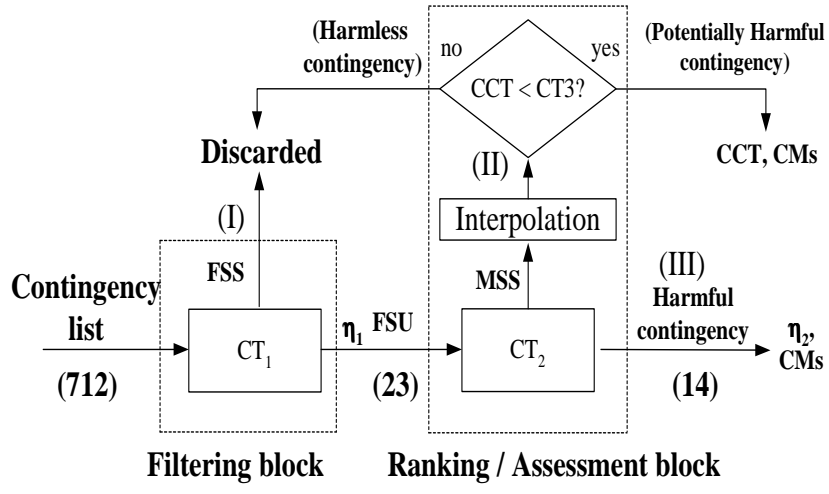

Fig. 2. Principle of operation for TSA Filtering and Ranking 
The continuous refreshing of the OMIB parameters by SIME allows to effectively identify the critical machines responsible for system transient instability. Within the OMASES TSA Application Function, SIME may also provide guidelines for stabilizing unstable cases by computing the excess power of critical machines and by sharing it among the non-critical ones. This is expected to provide insights for congestion management resolution.

\section{III.b) - VSA Voltage Stability Assessment}

Voltage dynamics in a power system is quite complex and several power system components and control devices may be involved in voltage instability/collapse phenomena. Voltage stability has been classified into short-term and long-term, according to the time frame involved. The representation of instantaneous dynamics (represented by algebraic constraints), short-term and long-term dynamics is necessary for a complete modeling [9].

The VSA Application Function integrates software tools for the assessment of Voltage Security into the DSA package of the OMASES platform. This includes, among others, the analysis of the impact of significant contingencies and the determination of secure operation limits in terms of power transfers in critical corridors or power consumption in load areas. For a given direction of stress and a given contingency, the Secure Operation Limit (SOL) of the system corresponds to the most stressed precontingency operating point such that the system responds to the contingency in acceptable way.

To meet the OMASES real-time goal, system analysis is based on the Quasi Steady State (QSS) approach for fast nonlinear simulation, together with linearization-based tools for diagnosis [9]. In long-term voltage stability studies in particular, the analysis concentrates on slowly varying components. Thus all short-term dynamics are considered as if at equilibrium; the long-term dynamics are described either by differential equations or discrete time equations.

The VSA function of OMASES consists of two modules: (1) the "Voltage Security Analysis Package" (VSAP) and (2) the "In-Depth Case Analysis Package" (IDCAP).

VSAP evaluates voltage stability security margins by means of a binary search. Further results of VSA are to display the power system area(s) in trouble for each contingency having a low security limit and to suggest preventive actions to maintain/increase security margins.

Contingency filtering is an important preliminary step also for VSA in order to identify harmless contingencies to be discarded from the security limit determination. To this purpose, contingencies are analysed at the maximum stress of interest with a properly tuned post-contingency power flow. Only those contingencies declared potentially harmful are analysed with the QSS time simulation [10]. The contingencies to be examined are usually all $(\mathrm{N}-1)$ cases (such as: loss of line, transformer, generator) to which a specified list of double $(\mathrm{N}-2)$ contingencies can be added.

IDCAP aim is to analyze in detail particular Voltage Stability scenarios, such as the system response to contingencies and load increase starting from the current operating point of the system or from an operating point retrieved from EMS historical data. For a given contingency (the default is the most constraining one calculated by VSAP) and direction of system stress, IDCAP evaluates the behavior of the system in the postcontingency configuration. The relative long-term simulation is performed using QSS approximation. IDCAP output consists of curves showing voltages versus regional load power for the affected region(s).

The user can easily apply additional disturbances to the default scenario, or simulate a different one.

The overall structure of the VSA function within OMASES is illustrated in Figure 3.

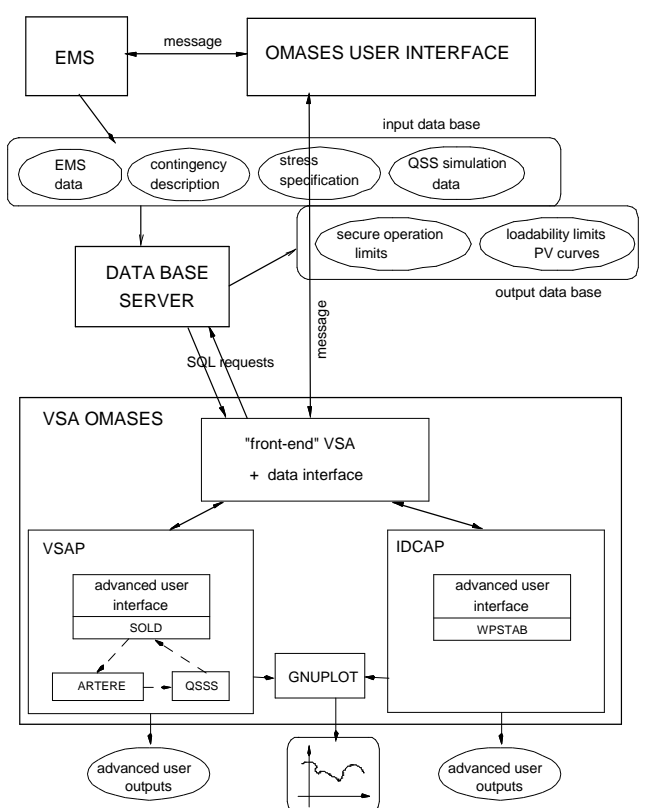

Fig. 3. VSA structure within OMASES

\section{III.c) - TS Training Simulator and MS Market Simulator}

The need for advanced training simulator for EMS operators is deeply felt, as dynamic phenomena such as cascade tripping, inter-area oscillations or voltage collapse are increasingly to be faced by operators. Restoration after major incidents is also a concern. Therefore training sessions must more and more integrate high-stressed and reduced-margin scenarios [11].

Within OMASES the TS is expected to run on-line or in the training mode. In the former case it has to play interactively any scenario starting from an actual operating point extracted from the EMS and taking into account any kind of disturbances or operator actions. In the latter case, the simulator must reproduce the real time, that is to say that an accurate simulated state of the system must be displayed at the operator station according to the refreshment time of the EMS (about 2 to $10 \mathrm{~s}$ ). To reach this twofold target, OMASES bases its developments on two different codes proprietary of Tractebel: EUROSTAG and FAST [11], [12]. 
The EUROSTAG program is a simulation tool developed jointly with EDF. The software computes the so-called "Extended Electromechanical Model" of the power system, valid in the range $(0-10 \mathrm{~Hz})$ (which corresponds to physical phenomena ranging from rotor transients up to daily load variation) and for large deviations from normal conditions (up to voltage or frequency collapse). The FAST program has been developed to serve as real time simulation engine for dispatcher training simulators. FAST runs basically the same model of the power system as the one of EUROSTAG, with some limitations in the complexity of the component models.

The market simulator is strictly related to the activity of the TS. In fact the active power dispatching of vertically organized utility systems is now substituted by the liberalized electricity market. The primary function of the MS within the OMASES environment is to build a generator dispatch for a defined future scenario. As previously expressed, the MS has to be adapted and tuned to the particular market structure it has to simulate. It is almost impossible to set-up a general purpose MS, thus in OMASES the TS and MS are specifically related to one experimental site - the Greek one, as better defined in the next section.

\section{III.d) - The site experimentation}

In order to test and validate the OMASES platform, two experimental sites have been made available by the Hellenic Transmission System Operator (HTSO) and by CESI. They respectively concern the EHV/HV Greek and Italian Transmission Systems. The validation, that has recently started, is expected to address the issues related to transient and voltage stability. The Greek site [3] is also testing the training simulator and the market simulator.

Within the experimentation phase, OMASES is going to be tested both in the Engineering mode (off-line) and in the Real-time mode (on-line). Two validation stages are thus prepared, respectively off-line and on-line.

In any case, an initial network situation and operating condition is made available directly from the EMS database or from a repository OMASES data base. The contingency list may consider $(\mathrm{N}-\mathrm{k})$ type of events, with the $(\mathrm{N}-1)$ usually acceptable. These may consist of single events or sequences of events such as faults on critical components, generation trip, protection misoperation, etc. Typical fault types (3-phase or single-phase) are to be considered, with specified fault clearing times. In particular, the following activities have to be tested: contingency filtering, ranking and evaluation (stable/unstable, margins), for a list of contingencies selected by system operators or, alternatively, for all the "N-1" type of contingencies originated by a snap-shot of the system provided by the EMS itself. Some ATC/TTC calculations are going to be carried out in the Engineering mode or in the Real-time mode for a number of source/sink patterns and the respective constraining conditions. The preventive control actions should provide guidelines to the operator for generation rescheduling and load-shedding [6].

\section{EXPERIMENTATION Phase: the ITALIAN Site}

The main objective of the Site Experimentation is to test the performances of the OMASES platform in a real world environment. Within the Italian experimentation site, OMASES is going to be tested both in the Real-time mode (on-line) and in the Engineering mode (off-line).

The Real-time mode is mainly concerned with the overall platform testing, and with the tool filtering/ranking capabilities. It is not likely that truly jeopardized situations occur, because the Italian power system is still operated in a conservative manner, with $\mathrm{N}-1$ - and often $\mathrm{N}-2$ - criterion being satisfied. It is expected that the implementation of the liberalized energy market may produce more stressed conditions.

The Engineering mode can be useful for a systematic validation of the tool capabilities and its tuning. Next, it may be interesting to check the output of the tool when heavily stressed scenarios are concerned. In the Engineering mode, if the initial conditions are "excessively" secure, the power system needs to be stressed by increasing the load, the power transfer between areas, weakening the topology and/or the generation capacity, creating low voltage conditions. The aim is to find the system limits and to compare suggested preventive actions with those used in the past or suggested by operator's experience.

\section{IV.a) - A reference for OMASES testing}

One aspect of the validation phase consists of comparing the simulation outputs of the TSA and VSA functions with a reference that represents the "real" system behavior. For the Italian system this reference is the electromechanical and long-term simulator SICRE, developed by CESI [13]. In particular, it is important that the models employed in the OMASES Application Functions be comparable to those implemented in SICRE.

The Application Functions TSA and VSA rely on different simulation packages. The TSA simulation engine is based on EUROSTAG, while the VSA package uses QSSS and WPSTAB. All of these simulators present their own modeling of the power system, and proper adaptations to reproduce modeling originally used in the Italian simulator are required.

A preliminary consideration concerns the time span that is going to be simulated: in the case of TSA, for instance, few seconds are sufficient, since experience suggests that multiswing phenomena do not occur in the Italian system. This allows neglecting the slow dynamics that a simulator takes into account, but which does not affect the outcome of the simulation (at least for the TSA aspects). Under such assumptions, for example, the boiler representation (steam plants) can be highly simplified, and LTCs can be assumed fixed. The final judgment whether a model simplification is acceptable relies, from a practical viewpoint, on the comparison of the simulation outputs obtained under identical conditions: to this aim, steady state and transient situations can be tested, such as short circuits, load steps, reference changes. EUROSTAG is a powerful simulator which in particular allows the user to build dynamic 
models of his own, integrated into the simulation. Process and control phenomena are implemented by means of such editable block diagrams, called macroblocks. A library of standard models is provided, and the user may adapt them or create quite new instances.

The most important models related to TSA concern the representation of synchronous machine, AVR, PSS, and turbine governor. Apart from the synchronous machine, the other models were built by means of macroblocks. Figure 4 shows EUROSTAG macroblocks reproducing the standard Italian AVR (bottom) and PSS (top) models. Proper adaptations were made to implement turbine governors.

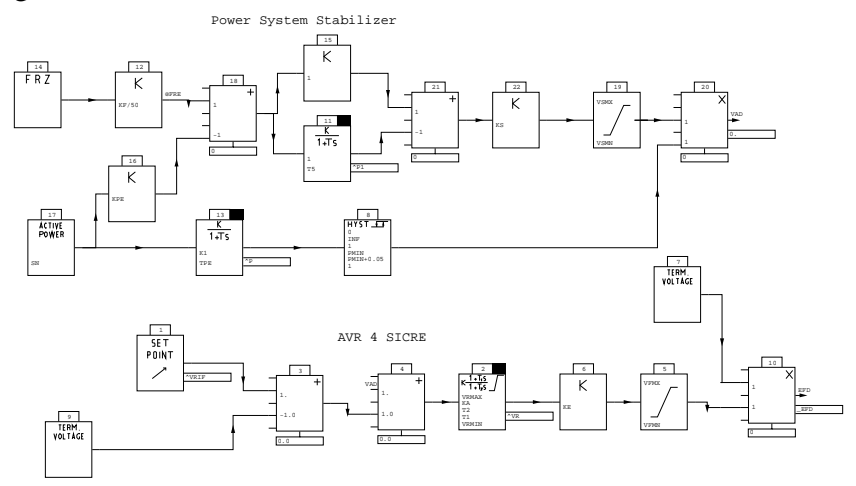

Fig. 4. Implementation of Italian AVR and PSS models into EUROSTAG

The VSA function relies on Quasi-Steady-State simulation, which is analogous to SICRE Long Term Dynamics (LTD) simulation mode. LTD activates itself when slow phenomena are concerned: electromechanical transients are considered as settled and the system can be described by a single average frequency, where only slow dynamics (boiler, LFC, ULTC, secondary voltage regulation...) are involved. In the course of a simulation, the program can switch automatically from the short term mode (with detailed electromechanical models) to the long-term mode. LTD integration step (typically $0.5 \mathrm{~s}$ ) and relevant models are quite close to those adopted by the OMASES VSA function.

\section{IV.b) - OMASES platform at the Italian site}

The Italian site is located at CESI (Milan), with remote connection to the Italian Transmission System Operator (GRTN - Gestore della Rete di Trasmissione Nazionale) based in Rome (Figure 5). The connection is made via ftp and updated every 15 minutes with the latest snapshot of the power system operating state (OMASES real-time mode).

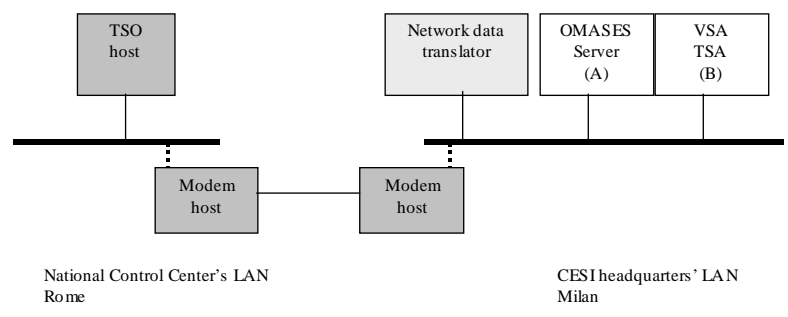

Fig. 5. OMASES configuration for CESI site experimentation
The data describing the Italian power system require preliminary translation from the Italian EMS format into OMASES format (Figure 6). A suitable bridge converts the power system data files into the required format and eventually sends them to the OMASES Real-time input directory. The Data Loader application loads the EMS data into the OMASES database, and a new analysis can start.

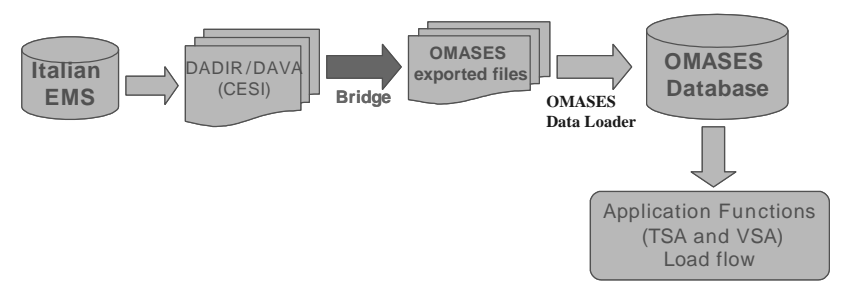

Fig. 6. EMS data translation and loading into OMASES database

\section{IV.c) - Italian system features and stress patterns for OMASES testing}

The Italian power system is characterized by a significant longitudinal structure which leads to the definition of critical interfaces. An instance of critical interface is the interconnection with central Europe, constituted by 15 tie lines of which 6 are $400 \mathrm{kV}$ lines and 9 are $220 \mathrm{kV}$ lines. The electrical energy importation is particularly important for Italy, and the interconnection is highly stressed. Moreover, also the internal North-to-South connections exhibit similar characteristics. The Florence-Rome interface, for instance, has six lines (five $400-\mathrm{kV}$ and one $220-\mathrm{kV}$ line) and the flow is normally from North to South. Figure 7 displays the network sections analyzed in detail with OMASES.

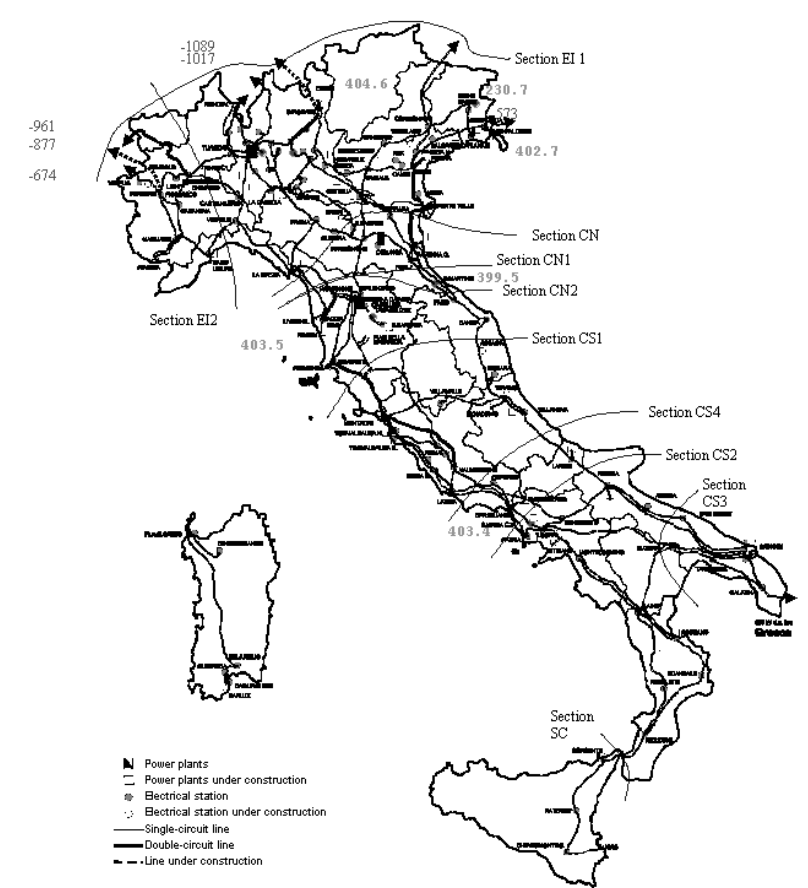

Fig. 7. One-line diagram of the 400-kV Italian system. Critical interfaces (sections) are highlighted. Reported numbers are OMASES VSA output 
This specific configuration of the Italian system constitutes a particular situation for the general security assessment problem fronted by DSA tools such as OMASES. The loss of a line in a critical interface may cause overloads or dynamic problems on the remaining lines. Then the corresponding scenarios not only need to be evaluated through contingency filtering and ranking, but suitable remedial actions have to be investigated [14]. A two-fold DSA activity can be envisaged, respectively $(A)$ "overall" addressed to security of the whole system - and $(B)$ "specific" - focused on critical interfaces.

For the Italian system the following three stress patterns are considered in the OMASES experimentation phase:

(a) Increase of interface power flow from the rest of Europe to Italy. Load is left unchanged in Italy, where generation is decreased. The interface is divided into three corridors and contingency filtering is applied. VSA limits may be compared with thermal overload limits and the associated risk of cascade line tripping leading to islanding, under-frequency load shedding or over-frequency unit tripping on the export side; (b) Increase of interface power flow between the Rome and Florence areas. A similar procedure, including contingency filtering, is followed; $(c)$ National load increase. Loads are increased according to patterns suggested by past situations.

\section{IV.d) - TSA scenarios for the Italian system}

Past experience and extensive simulation show that transient instability has rarely been an issue in the Italian system, at least as long as it has been operated in the "monopolistic fashion". The TSA function is still under preliminary set up. As for the Engineering mode, a testing of the TSA function requires to devise heavily stressed scenarios, such as the one presented hereafter that describes two cases of disturbances on the CenterSouth-South interface and the Southern area.

The different phases composing the scenario are listed. The base case is extracted from the EMS repository database.

\section{(i) Preliminary network weakening}

- Opening of the $380 \mathrm{kV}$ line Latina-Garigliano

- Opening of the $380 \mathrm{kV}$ line Ceprano-Garigliano

- Opening of the $220 \mathrm{kV}$ line Popoli-Capriati

(ii.1) Contingencies (case 1)

- Permanent 3-phase short circuit on the $380 \mathrm{kV}$ line Presenzano-Valmontone line, at a $10 \%$ distance from Presenzano

- Opening of the line at both ends after $0.5 \mathrm{~s}$ from short circuit inception

(ii.2) Contingencies (case 2)

- Permanent 3-phase short circuit on the $380 \mathrm{kV}$ line Presenzano-Valmontone, at a $10 \%$ distance from Presenzano

- Opening of the line at both ends after $0.2 \mathrm{~s}$ from fault inception

- Opening of the $380 \mathrm{kV}$ line Larino-Villanova after 0.5 seconds - System separation

\section{(iii) Outcome}

In case (1) one of the Presenzano units loses synchronism. If the group is then disconnected (simulating the intervention of its protection) the network reaches a stable state. Case (2) results in grid separation. Moreover, the generators in the South slow down with respect to Sicilian ones. In absence of any intervention, loss of synchronism and load shedding take place.

\section{PReliminary Results}

This Section presents some outputs of the OMASES VSA function. The results were obtained in the early testing phase of the Real-time mode of operation. This phase was performed with the objective of running the platform, rather than obtaining accurate results that are expected later on, in the sequel of the testing. In fact the VSA dynamic parameters used in the simulations still need some tuning (unlike the load flow data, which correspond to actual system conditions). Some results are presented, also with the aim of showing the output layout of the VSA function. Analogous simulations and results are presented in [3].

Early results with the VSA function concern the secure operation limits of the system when it undergoes a "uniform" load stress. Under this pattern, the maximum stress of interest is a load increment of $5000 \mathrm{MW}$, distributed throughout the system ("IZone 3"). The corresponding maximum generation increment is set at $1020 \mathrm{MW}$ for the generators in Milan area ("gZone l", North of the country), the remaining load demand being covered by all generating units according to their speed droop. This choice was suggested by previous studies, and results in an increased power flow from North to South.

During the first investigations, all $\mathrm{N}-1$ and $\mathrm{N}-2$ contingencies involving line outage were taken into account, for the interfaces ("sections") highlighted in Figure 7. Such interfaces were identified as potentially critical by past experience and by extensive simulation, both static and dynamic.

The reported results concern to two daytime situations and a night-time situation for a weekday and a weekend day.

The first day presented is Wednesday, 5 March 2003, with analyses performed for the situations at about 00.15 AM and 10.11 AM. The second day considered is Saturday, 15 March 2003, at the same times. The analyses performed on the cases $12 \mathrm{PM}$ and $6 \mathrm{PM}$ are similar to the morning cases and will not be described in detail.

Figures 8 and 9 report the output of ASTRE (VSAP package) in terms of secure operation margins (MW). The bar chart displays the margin associated with each contingency, as results from the binary search. The search is performed by simulating the contingency under several stress conditions, in between the base case (no stress) and the maximum stress of interest, according to the binary search method. The figures show the last two stress levels for each contingency: the "last accepted" value is the maximum stress level that resulted in an acceptable postfault system evolution; the "first refused" value 
corresponds to the minimum stress level, whose post-fault evolution was rejected. An operating condition presenting zero margin with respect to a contingency is not secure even in the unstressed state, i.e., its response to the contingency is not acceptable even at zero stress (base case). The values in the bar chart refer to the simulated load increase, while the imposed generation increase in the gZone 1 is computed proportionally.

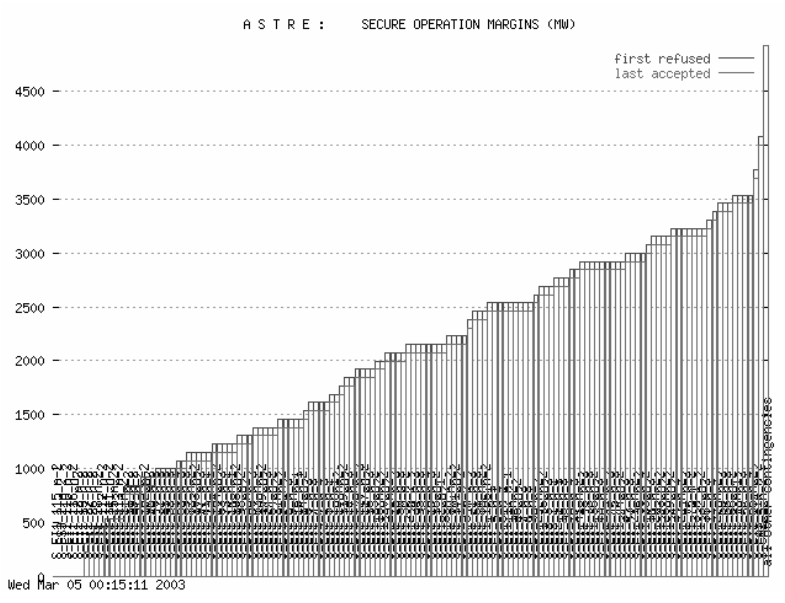

Fig. 8. Secure Operation Margins computed by VSAP for the situation Wednesday, 5 March 2003 at 00.15 AM

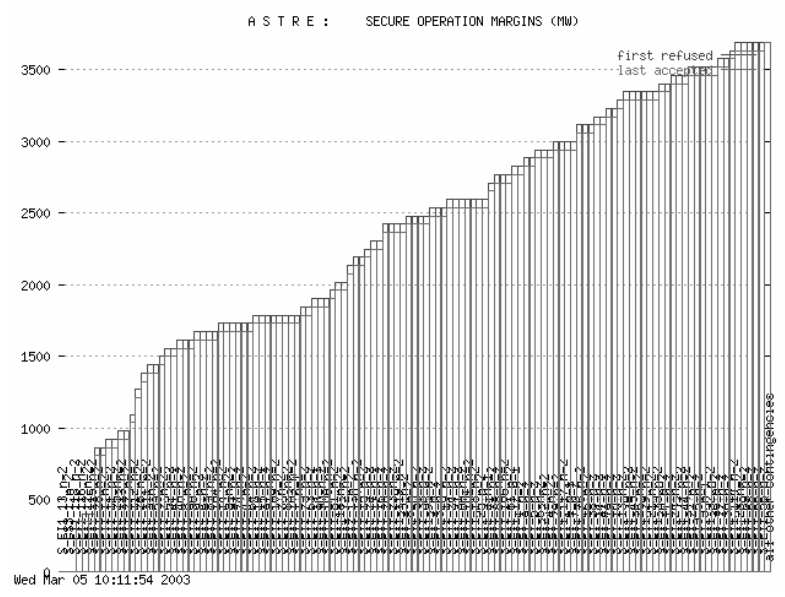

Fig. 9. Secure Operation Margins computed by VSAP for the situation Wednesday, 5 March 2003 at 10.11 AM

If the operator wants to keep the system secure with respect to all of the analysed contingencies, the maximum allowable stress must be within the minimum margin provided by the analysis. If such value is associated to a contingency which is not likely to occur (e.g. a double outage), or whose effects are limited to a small part of the system only, then the operator may choose to implement such stress and accept the risk of instability.

The security margins are also reported in tables (as in Figure 10), where the contingencies are ranked according to the respective severity. In all cases, the first-ranking contingencies have names ending by "N-2", corresponding to double outage events. In some cases the margin is zero, i.e., one or more contingencies may jeopardise even the current operating state; but they are all (rather unlikely) "N-2" contingencies, no "N-1" being involved. This means the system is quite robust.

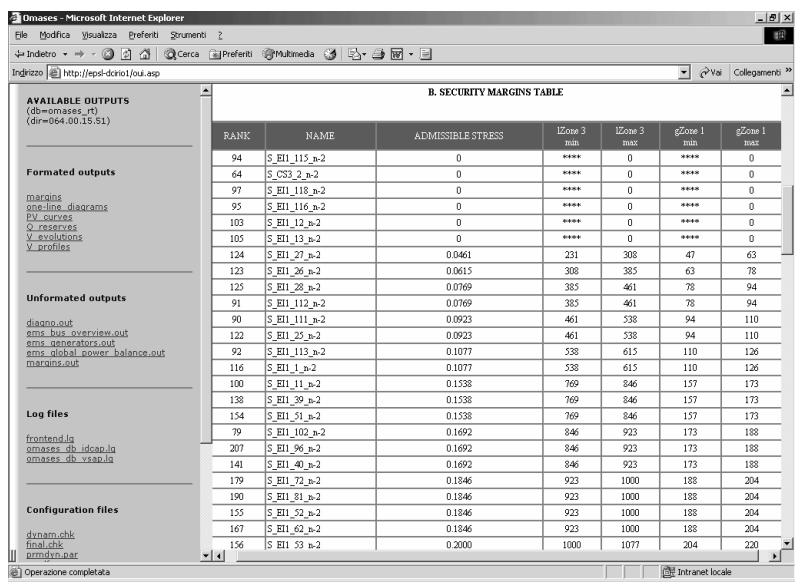

Fig. 10. Security margins table, referred to the situation Wednesday, 5 March 2003 at 00.15 AM

The column "Admissible stress" reports the proportion of the specified maximum stress, the contingency allows to implement (range in between 0 and 1). Next columns present the system stresses at the borderline cases, for both load (cf. the bar chart) and generation: "IZone 3 min" is the upper feasible load increment, "IZone 3 max" the first unfeasible. The same holds for generation. For example, let's consider the mentioned weekday case at midnight. Six contingencies have no margin: they are all N-2 contingencies affecting the interconnection with Central Europe or (in one case) the interface CS3, Southeast of Italy, where large power plants are installed with few transmission lines available. The first contingency having a positive margin is contingency number 124 . It has an admissible stress of $231 \mathrm{MW}=0.0461$ ("normalised" admissible stress) * $5000 \mathrm{MW}$ (max stress). Accordingly, the generation increase in gZone 1 is $47 \mathrm{MW}=0.0461 *$ $1020 \mathrm{MW}$ ( $\max$ imposed generation increment).

In the weekend day at the same time there are five contingencies with no margin, all of the N-2 type. They regard the same interfaces as in the previous case. The first contingency with positive margin presents an admissible stress of $472 \mathrm{MW}$. By comparing the results relative to these two situations, it emerges that the weekday case has smaller margins (for instance, the number of contingencies with a margin smaller than $1000 \mathrm{MW}$ is larger in the first case).

When comparing the analyses of Wednesday, 5 March at $00.15 \mathrm{AM}$ and at 10.11 AM, an unexpected result emerges: the daytime case has larger secure operation limits than the night-time case. This may due to the current practice of generator shut-down at night: voltage is no more supported, and the system is weaker. This situation did not occur in the past, when many generators were not disconnected, they simply worked at low power.

Another output provided by VSAP is the voltage profile of the first refused stress level (Figure 11). Voltages are 
arranged in increasing order. The resulting histogram therefore represents the number of buses whose voltage is lower than the level of the considered bus. A steep profile, with few voltage depressed buses, denotes local voltage problems; conversely, a "softer", low voltage profile may indicate wider-extent phenomena.

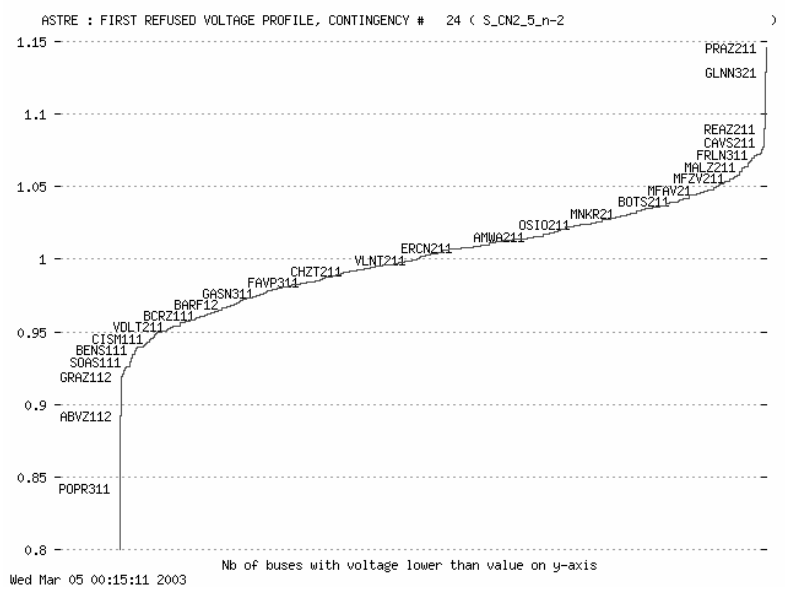

Fig. 11. First refused voltage profile for contingency no. 24. Buses are arranged according to an increasing voltage magnitude order (p.u. of the rated value of each bus). Case Wednesday, 5 March 2003 at 00.15 AM

Once again, the daytime case seems better than the nighttime one. In fact the voltage increases steadily to "robust" values (0.95 p.u. and above) in the $10.11 \mathrm{AM}$ situation; the profile is instead lower in the midnight case. The weekend day night profile appears to be good.

A third important result of the VSAP package is the plots of bus voltage vs. time. For each contingency a plot shows the time evolution of the "worst" bus voltage. The initial steady state is simulated up to 10 seconds, then the contingency is applied. The program goes on simulating till $900 \mathrm{~s}$ (15 minutes), unless the QSS simulation encounters a singularity (loss of equilibrium of short-term dynamics) or it is stopped due to violation of dynamic criteria.

The last output of VSAP-ASTRE concerns the reactive reserves and it is shown in Figure 12.

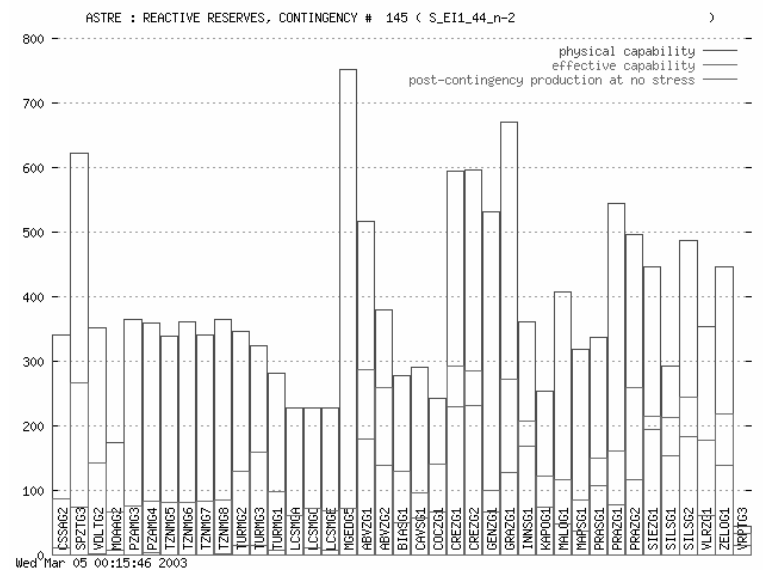

Fig. 12. Reactive reserves for contingency no. 145. Case Wednesday, 5 March 2003 at 00.15 AM
For each contingency a bar chart reports the reactive production and limits of the most stressed generators by contemporaneously indicating the post-contingency reactive production at no stress, the effective capability and the physical capability.

\section{CONCLUSIONS}

The paper has presented the structure, methodologies and assumptions adopted in a research project on Dynamic Security Assessment, partially supported by EU. The experimental site concerning the Italian system is described and preliminary results are reported. Results of the experimental process are permitting the tuning and the validation of the DSA platform in terms of experience and feed-backs for what concerns the effective possibilities of filtering, screening and stabilizing contingencies in large electrical power systems.

\section{REFERENCES}

[1] Proceedings of the IEEE, Special Issue on the Technology of Power System Competition, Vol. 88, No. 2, February 2000

[2] OMASES - Open Market Access and Security Assessment System, EU Contract N. ENK6-CT2000-00064, December 2000

[3] C. D. Vournas, G. A. Manos, J. Kabouris, G. Christoforidis, G. Hasse, T. Van Cutsem, "On-Line Voltage Security Assessment of the Hellenic Interconnected System”, IEEE Bologna Powertech 2003 Conference, Bologna (Italy), June 23-26, 2003

[4] F.A. Rahimi, N.J. Balu, M.G. Lauby, "Assessing on-line Transient Stability in Energy Management Systems", IEEE Computer Applications in Power, Vol. 4, No.3, July 1991, pp. 44-49

[5] K. Demaree, T. Athay, K. W. Cheung , Y. Mansour, E. Vaahedi, A.Y. Chang, B.R. Corns, B.W. Garrett, "An On-line Dynamic Security Analysis System Implementation", IEEE Transactions on Power Systems, Vol. 9, No. 4, November 1994, pp. 1716-1722

[6] A. Bihain, G. Burt, F. Casamatta, T. Koronides, R. Lopez, S. Massucco, D. Ruiz Vega, C. Vournas, "Adavanced perspectives and implementation of dynamic security assessment in the open market environment", 39-101, CIGRE General Session, Paris, August 2002

[7] M. Pavella, D. Ernst, D. Ruiz-Vega, "Transient Stability of Power Systems - A unified approach to assessment and control", Kluwer's Power Electronics and Power Systems Series, October 2000

[8] M. Pavella, L. Wehenkel, Y. Zhang, "SIME: A hybrid approach to fast transient stability assessment and contingency selection", Electric Power \& Energy Systems, Vol. 19, No. 3, 1997, pp. 195-208

[9] T. Van Cutsem, C. Vournas, "Voltage Stability of Electric Power Systems", Kluwer Academic Publishers (Power Electronics and Power Systems Series), Boston, 1998

[10] T. Van Cutsem, F. Capitanescu, C. Moors, D. Lefebvre, V. Sermanson, "An advanced tool for preventive voltage security assessment", Proc. 7th SEPOPE, Curitiba (Brazil), May 2000

[11] S. Gissinger, P. Chaumes, J.-P. Antoine, A. Bihain, M.Stubbe: “Advanced Dispatcher Training Simulator", IEEE Computer Applications in Power, Vol. 13, No. 2, 2000, pp. 25-30

[12] M. Stubbe, A.Bihain, J.Deuse, J.C. Baader, "STAG - A New Unified Software Program for the Study of the Dynamic Behaviour of Electrical Power Systems", IEEE Transactions on Power Systems, Vol. 4, No 1, 1989, pp.129-138

[13] P. Baratella, P. Scarpellini, R. Marconato, B. Cova, E. Gaglioti, R. Zacheo, "A Power System Simulator Covering Different Time Scale Phenomena: Models, Algorithms, MMI and Test Results", Proc. IEEE Stockholm Power Tech, Stockholm, June 1995, pp. 376-381

[14] D. Cirio, S. Massucco, A. Morini, F. Silvestro, L. Polese, R. Salvati, F. Casamatta, G. Demartini, P. Scarpellini, "A decision support system for preventing power system degradation into emergency conditions", IEEE ISAP 2001 Conference, Budapest, June 18-21, 2001 